\title{
CARACTERÍSTICAS CLÍNICO-PATOLÓGICAS Y SOBREVIDA DE PACIENTES CON CÁNCER DE MAMA BILATERAL SINCRÓNICO*
}

\author{
Drs. César Sánchez R. ${ }^{1}$, Francisco Acevedo C. ${ }^{1}$, Militza Petric G. ${ }^{2}$, \\ Francisco Domínguez C. ${ }^{2}$, Augusto León R. ${ }^{2}$, Dravna Razmilic V. ${ }^{3}$, \\ Als. Carolina Ceballos B. ${ }^{4}$, Fernando Espinoza C. ${ }^{4}$, \\ Drs. M. Elena Navarro O. ${ }^{3}$, David Oddó B. ${ }^{5}$, Mauricio Camus A. ${ }^{2}$
}

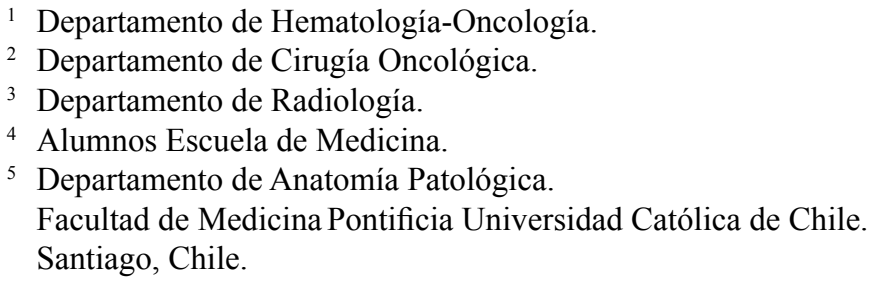

\section{Abstract \\ Clinicopathological features and survival of patients with bilateral synchronous breast cancer}

Introduction: Bilateral synchronous breast cancer (BSBC) has a variable incidence and conflicting data on prognosis. Our goal is to evaluate the characteristics and survival of patients with BSBC treated at Cancer Center of the Pontificia Universidad Católica de Chile. Patients and Methods: Descriptive study. We identified patients treated between January 1999 and May 2013. We evaluated and compared characteristics from BSBC patients with a local cohort of non-synchronous breast cancer. Results: The incidence of BSBC was $1.8 \%(n=28)$. Three patients were excluded because of missing data. Therefore we analyzed 25 patients (50 tumors). Median age: 50 years (33-84). The dominant tumor's diagnosis was clinical in $47.6 \%$ of cases, and imaging in $90.5 \%$ of the contra-lateral non-dominant $(\mathrm{p}=0.04)$. Average tumor size of invasive tumors was $2.5 \mathrm{~cm}$ for dominant tumor versus $1.7 \mathrm{~cm}$ for the non-dominant $(\mathrm{p}=0.02) .81 .4 \%$ of invasive tumors were estrogen receptor (ER) positive. Histological and subtype concordance between both tumors was $88 \%$ and $72 \%$ respectively. $54 \%$ of patients with $\mathrm{BSBC}$ were managed with total mastectomy versus $28.4 \%$ in the control group no BSBC $(p=0.0001)$. The estimated overall survival at 10 years was $76.5 \%$ in patients with no BSBC versus $62 \%$ in those with BSBC $(p=0.08)$. Conclusions: The BSBC is rare. Frequently they are ER positive tumors, mostly diagnosed through images and managed with less conservative surgery.

Key words: Breast cancer, synchronic, bilateral, prognosis.

*Recibido el 6 de octubre de 2013 y aceptado para publicación el 18 de noviembre de 2013.

Los autores no refieren conflictos de interés.

Correspondencia: Dr. César Sánchez R. csanchez@med.puc.cl 


\section{Resumen}

Introducción: La incidencia y pronóstico del Cáncer de mama (CM) bilateral sincrónico (CMBS), no son bien conocidos. Evaluamos las características y sobrevida de pacientes con CMBS tratadas en el Centro de Cáncer de la Pontificia Universidad Católica de Chile. Pacientes y Método: Estudio descriptivo. Identificamos pacientes con CMBS tratadas entre enero de 1999 y mayo de 2013. Evaluamos sus características y las comparamos con una cohorte local de CM no sincrónico. Resultados: La incidencia de CMBS fue de 1,8 $\%(\mathrm{n}=28)$. Se excluyeron 3 pacientes por falta de datos. Analizamos por tanto, 25 pacientes (50 tumores). Mediana de edad: 50 años (33-84). El diagnóstico del tumor dominante fue clínico en el 47,6\% de los casos, y por imágenes en $90,5 \%$ de los contra-laterales no dominantes $(\mathrm{p}=0,04)$. Tamaño tumoral promedio de los tumores invasores fue de $2,5 \mathrm{~cm}$ en tumores dominantes vs $1,7 \mathrm{~cm}$ en los no dominantes $(\mathrm{p}=0,02)$. Un $81,4 \%$ de los tumores invasores fueron receptor de estrógenos (RE) positivos. La concordancia histológica y de subtipo histológico entre ambos tumores fue de $88 \%$ y $72 \%$ respectivamente. Un $54 \%$ de las pacientes con CMBS fueron manejadas con mastectomía total versus un $28,4 \%$ en el grupo control de CM no BS $(\mathrm{p}=0,0001)$. La sobrevida global estimada a 10 años fue de un $76,5 \%$ en pacientes con CM no BS versus $62 \%$ en aquellos con CMBS $(\mathrm{p}=0,08)$. Conclusiones: El CMBS es poco frecuente. Usualmente son tumores $\mathrm{RE}$ positivos que reciben con menos frecuencia manejo conservador de la mama.

Palabras clave: Cáncer de mama, bilateral, sincrónico, pronóstico.

\section{Introducción}

El CM es la primera causa de muerte en mujeres chilenas ${ }^{1}$. Luego del diagnóstico de $\mathrm{CM}$, el riesgo de desarrollar CM contra lateral es 2 a 6 veces mayor que en la población sin $\mathrm{CM}$ previo ${ }^{2}$. El aumento de tumores diagnosticados en forma precoz, el estudio detallado con imágenes de la mama y mejor sobrevida pueden explicar un aumento de la frecuencia de tumores contra-laterales en algunas series ${ }^{2,3}$.

Mientras la incidencia de CM bilateral varía entre el 1,4 al $12 \%$, el CMBS tiene una incidencia de 1 a $3 \%{ }^{3}$. El valor pronóstico de esta forma de presentación, sus bases biológicas y su historia natural no están claramente definidas ${ }^{3,4}$. Nuestro objetivo es mostrar las características clínicas de una cohorte de pacientes con CMBS tratadas en nuestra institución, describir sus características patológicas, comparar el fenotipo del tumor primario y el contra lateral, y evaluar su sobrevida en relación a una cohorte de pacientes con $\mathrm{CM}$ no bilateral sincrónico.

\section{Pacientes y Método}

Este es un estudio descriptivo. Identificamos pacientes con CMBS tratadas en el Centro de Cáncer, de la Pontificia Universidad Católica de Chile, entre enero del año 1999 y mayo del año 2013. Se excluyeron pacientes con etapa metastásica al debut. Definimos como CMBS aquel que se presentaba con tumor contra lateral, invasor o in situ, menos de 3 meses luego del diagnóstico del tumor primario, pesquisado clínicamente o por estudio radiológico ${ }^{3}$. Llamamos tumor sincrónico dominante al tumor con la etapa más avanzada, o, en los casos en que ambos tumores tenían la misma etapa, al tumor más grande.
Se registró: edad, tipo de cirugía (mastectomía total versus parcial), hallazgos patológicos del tumor, sobrevida global (SG) y específica por la enfermedad (SEE). La SG fue definida como el tiempo transcurrido desde la biopsia inicial diagnóstica hasta el fallecimiento por cualquier causa. La fecha y causas de muerte se obtuvieron de datos de los certificados de defunción obtenidos del registro civil y de registros en nuestra base de datos clínica. El análisis patológico incluyó: tipo histológico, grado de diferenciación según la clasificación de Elston y Bloom, modificada por Elston y Ellis ${ }^{5}$, estudio de receptor estrogénico (RE) y receptor de progesterona (RP), en escala de $0-100 \%$, receptor de factor de crecimiento epidérmico tipo 2 (HER 2) escala de + $\mathrm{a}+++$. Todos por métodos de inmunohistoquímica (IHC). Siendo para HER2: + negativo, ++ incierto evaluado adicionalmente por inmunofluorescencia in situ (FISH) y +++ positivo. Se definió el subtipo tumoral clínico-patológico según los siguientes elementos: Luminal A (RE + y/o RP +, grado histológico (GH ) 1-2, HER2 negativo); Luminal B (RE + y/o $\mathrm{RP}+$, GH 3 y /o HER 2 positivo), Triple negativo (TN) (RE -, RP -, y HER negativo), HER2+ (RE - y RP -, HER 2 positivo) ${ }^{6}$. Para la SEE se definió el mismo período que la SG y se contabilizó sólo las pacientes que fallecen de CM.

Comparamos características clínicas, tipo de manejo quirúrgico, hallazgos patológicos, SG y SEE de CMBS con una cohorte de nuestro centro de pacientes con $\mathrm{CM}$ no bilateral tratadas en la misma época. Analizamos los datos con programa SPSS21.0, utilizando estadística descriptiva. Calculamos la sobrevida según Kaplan-Meier y se compararon las curvas con test de Log-Rank. Consideramos diferencias estadísticamente significativas $\mathrm{p}<0,05$. 


\section{Resultados}

Registramos 1.523 pacientes con CM en etapas I a III durante el período. De ellos, 28 corresponden a CMBS, con una prevalencia para esta última presentación de un 1,8\%. Se excluyeron 3 pacientes: datos incompletos de registro y/o seguimiento (2), actualmente en tratamiento (1). Analizamos por tanto, 25 pacientes (50 tumores), todas mujeres. Las características de las pacientes se describen en Tablas $1 \mathrm{y}$ 2. Mediana de edad: 50 años (33-84). La sospecha diagnóstica del tumor dominante fue inicialmente clínica en el 47,6\% (11/21) de los casos. En contraste, el tumor no dominante fue diagnosticado clínicamente en el 9,5\% $(2 / 21)$ de las pacientes $(p=0,04)$. En 4 pacientes no disponemos de esta información. En comparación con los tumores no dominantes, los dominantes, fueron más frecuentemente manejados con mastectomía total $(54 \%$ versus $28,4 \%$, $\mathrm{p}=<0,0001)$ y disección axilar $(72,0 \%$ vs $37,5 \%$, $\mathrm{p}=0,04)$. Del total de tumores $43 / 50(86 \%)$ eran invasores y $7(14 \%)$ eran carcinomas in situ $(14,0 \%)$. $\mathrm{Al}$ analizar los tumores invasores, aquellos dominantes fueron de mayor tamaño que su contraparte no dominante: mediana de tamaño tumoral de 2,5 $\mathrm{cm}$ versus $1,7 \mathrm{~cm}$ respectivamente $(\mathrm{p}=0,02)$. En los tumores invasores el tipo histológico más frecuente fue carcinoma ductal $(62,5 \%)$, seguido de carcinoma lobulillar (18\%); además el $81,4 \%$ (35/43) eran RE positivos, un caso $(2,3 \%)$ fue HER2 positivo. De acuerdo a nuestra definición de subtipos clínicopatológicos (ver sección material y métodos) en el total de tumores invasores los subtipos histológicos fueron: Luminal en 35/43 casos (44,2\% Luminal A; $34,9 \%$ Luminal B; $2,3 \%$ Luminal no especificado) y TN en $8 / 43$ casos $(18,6 \%)$. No encontramos tumores del subtipo HER-2 positivo (HER-2 positivo y RE, RP negativos). En pacientes con tumores sincrónicos invasores, la concordancia histológica y de subtipo histológico entre el tumor dominante y no dominan-

Tabla 1. Características clínico-patológicas de 25 pacientes (50 tumores) con cáncer de mama bilateral sincrónico

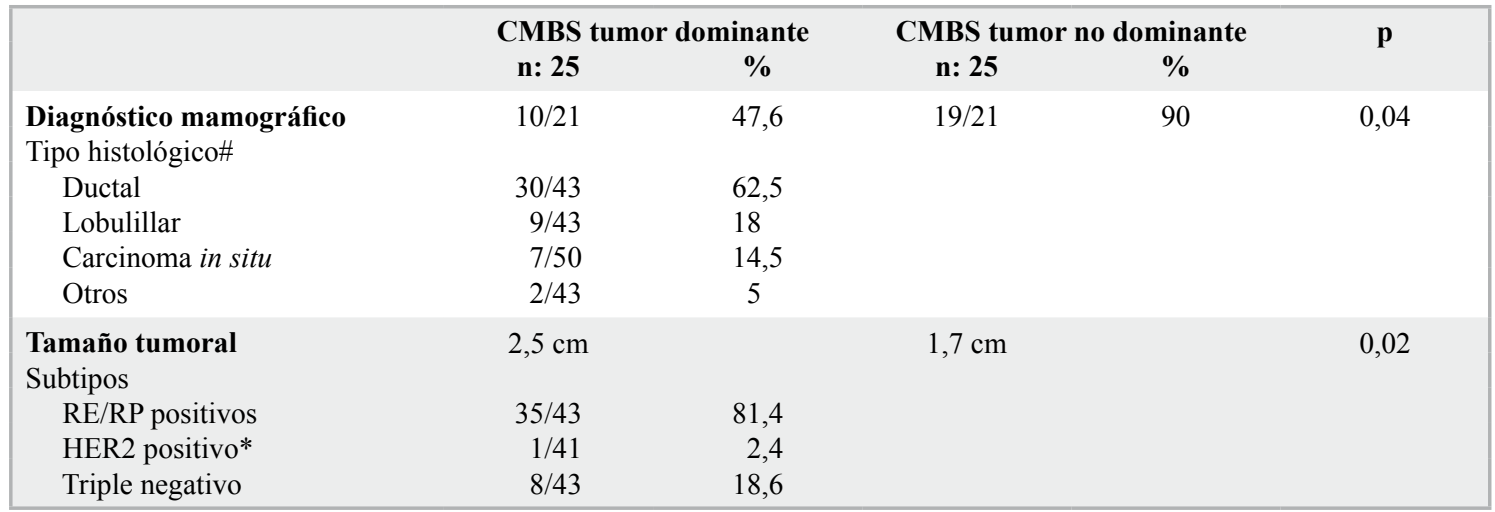

*HER 2 positivos independiente del estado de RE. ${ }^{\# 2}$ tumores sin histología específica conocida.

Tabla 2. Comparación de 25 pacientes con cáncer de mama sincrónico bilateral y 1.495 pacientes con cáncer de mama no bilateral sincrónico

\begin{tabular}{|c|c|c|c|c|c|}
\hline & & & & & \\
\hline & $\mathbf{n}$ & $\%$ & $\mathbf{n}$ & $\%$ & \\
\hline & 25 & $1,8 \%$ & 1.495 & $98,2 \%$ & \\
\hline Mediana edad (rango) & $50(33-84)$ & & $55(25-95)$ & & $\mathrm{p}=0,57$ \\
\hline Mastectomía total & $27 / 50$ & $54,0 \%$ & $369 / 1.300^{\#}$ & $28,4 \%$ & $\mathrm{p}=<0,0001$ \\
\hline Histología lobulillar & $9 / 43^{*}$ & $20,9 \%$ & $112 / 1.467$ & $7,6 \%$ & $\mathrm{p}=0,002$ \\
\hline SG a 10 años & & $\begin{array}{c}62,0 \% \\
( \pm 13,2)\end{array}$ & $\begin{array}{l}76,5 \% \\
( \pm 1,7)\end{array}$ & & $\begin{array}{c}\mathrm{p}=0,08 \\
(\log \text { Rank) }\end{array}$ \\
\hline SEE a 10 años & & $\begin{array}{l}86,2 \% \\
( \pm 5,3)\end{array}$ & & & \\
\hline
\end{tabular}

*Se excluyeron los tumores in situ en el análisis. "Se excluyeron 167 pacientes por falta de datos. 


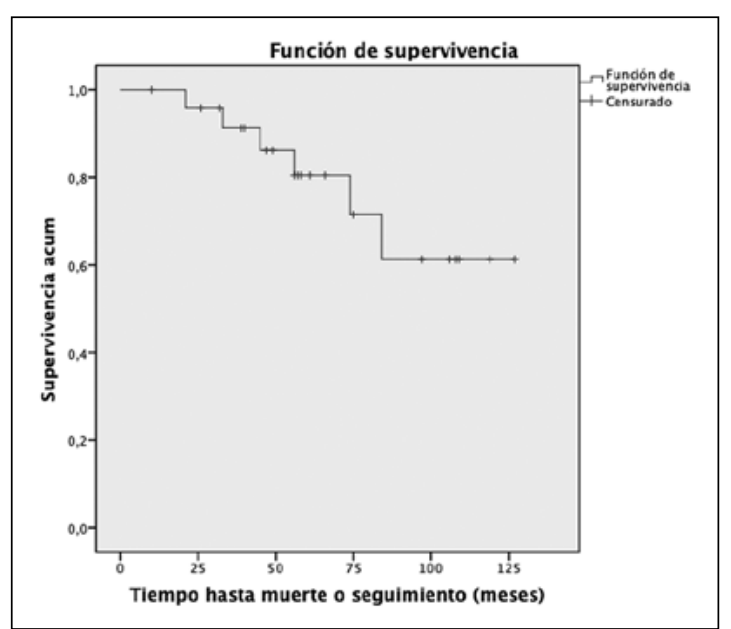

Figura 1. Sobrevida global de una cohorte de pacientes con cáncer de mama bilateral sincrónico, tratados en el Centro de Cáncer, Pontificia Universidad Católica de Chile entre el año 1999-2013.

te, fue de $88 \%$ y $72 \%$ respectivamente. La SG de pacientes con CMBS a 5 y 10 años fue de $79,7 \%$ y $62 \%$ respectivamente (Tabla 2, Figura 1). Mientras la $\mathrm{SG}$ de nuestro grupo control con $\mathrm{CM}$ no BS a 5 y 10 años fue de $90,2 \%$ y $76,5 \%$ respectivamente, superior al grupo con CMSB, esta diferencia no fue estadísticamente significativa $(\mathrm{p}=0,08)$ (Tabla 2 , Figura 2). Con una mediana de seguimiento de 57 meses (10-127 meses), 6 pacientes han fallecido, 3 por CM. SEE estimada a 10 años de $86,2 \%$ (Tabla 2 , Figura 3). La SEE a 10 años en el grupo de pacientes con CMBS fue de un $86,2 \%$ (Tabla 2). No disponemos de datos fiables en el seguimiento acerca de SEE en nuestra cohorte de pacientes con CM no BS.

\section{Discusión}

La incidencia de CM bilateral es, en la mayoría de las series reportadas, entre el 1,4 al 20\% de los casos, dependiendo del método de estudio de la mama contra lateral y la longitud del seguimiento ${ }^{2,7}$. Mujeres diagnosticadas con CM tienen un riesgo mayor de desarrollar enfermedad contra lateral, y de los factores asociados a este riesgo, la edad parece ser el más importante ${ }^{2,3}$. Mujeres con diagnóstico de $\mathrm{CM}$ entre los 30 y 39 años tienen $15-25$ veces mayor riesgo de desarrollar enfermedad contra lateral, entre los 40-49 años este riesgo es 5 veces mayor, y entre los 50-59 es 2,5 veces superior. El riesgo para una mujer sobre los 60 años es similar al de la población general ${ }^{8}$. Otros factores que influyen en el desarrollo CM contra lateral son: historia familiar de CM,

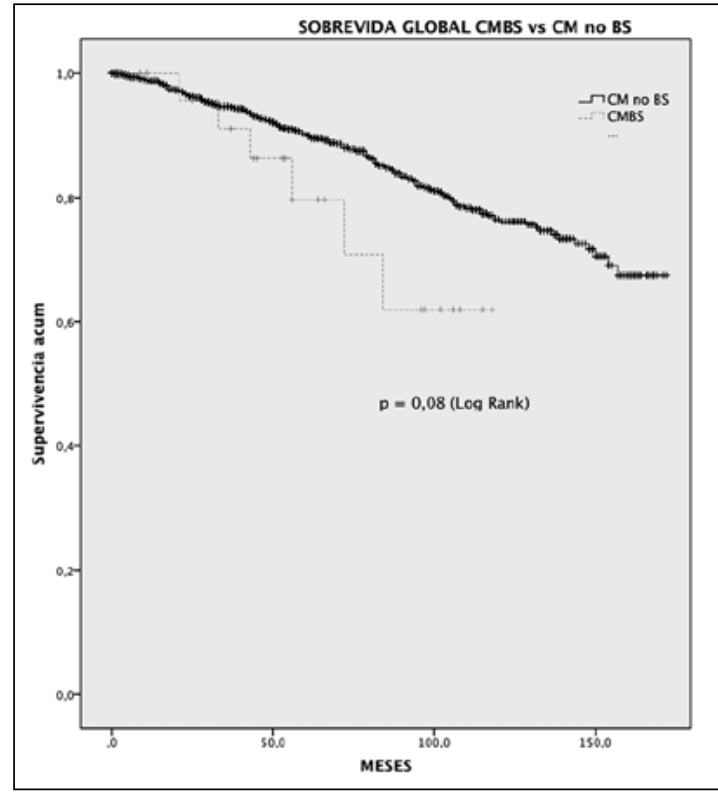

Figura 2. Sobrevida global de una cohorte de pacientes con cáncer de mama bilateral sincrónico, comparado con una cohorte de pacientes con cáncer de mama no bilateral sincrónico, tratados en el Centro de Cáncer, Pontificia Universidad Católica de Chile entre el año 1999-2013.

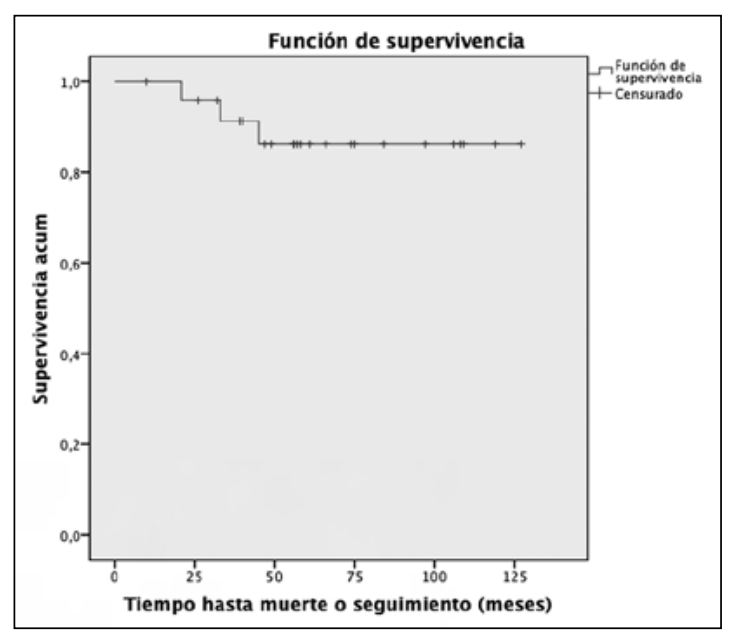

Figura 3. Sobrevida específica por enfermedad de una cohorte de pacientes con cáncer de mama bilateral sincrónico, tratados en el Centro de Cáncer, Pontificia Universidad Católica de Chile entre el año 1999-2013.

histología lobular, nuliparidad, multicentricidad del primario y tratamiento adyuvante recibido ${ }^{2}$.

El CMBS representa entre el 0,2 al 3,2\% de todos los casos nuevos diagnosticados ${ }^{2-4}$. Esta variación en la incidencia depende de su definición, época del estudio, inclusión de histologías ductales, lobu- 
lares o neoplasias in situ. En nuestra experiencia la incidencia de CMBS fue de un $1,8 \%$, una cifra de acuerdo con la mayoría de los reportes, definiendo CMBS como aquellos tumores invasores (excluyendo neoplasias in situ) diagnosticados dentro de 3 meses del primario.

El estudio de la mama con resonancia magnética pesquisa un $3 \%$ de tumores contra laterales, no encontrados por mamografía o ecografía mamaria. Mientras se acepta que la resonancia magnética es un examen muy sensible para la detección de enfermedad oculta, su uso en el ámbito preoperatorio es variable y controversial ${ }^{9}$. A pesar de la creencia de que la cirugía, planificada con resonancia, puede mejorar los resultados quirúrgicos, mejorando los márgenes y permitiendo una extirpación más segura de la enfermedad macroscópica, no se ha demostrado aun su beneficio clínico en el largo plazo. La controversia de su uso también está dada por el aumento en los niveles de mastectomía y escisiones amplias para tratar la posible enfermedad residual, que de otro modo sería controlada por cirugía conservadora estándar y radioterapia ${ }^{10}$.

El manejo del CMB es aún incierto. Mientras las pacientes pueden ser tratadas con cirugía conservadora, hay un aumento en los niveles de mastectomías totales en esta presentación clínica ${ }^{11}$. Esto puede ser por la percepción de mayor riesgo asociado a enfermedad bilateral, y costos financieros asociados al seguimiento y tratamiento de la mama contra lateral ${ }^{12}$. En nuestra serie el nivel de mastectomía total fue mayor en el grupo con CMBS (54\%) que en nuestra cohorte de $\mathrm{CM}$ no bilateral sincrónico $(28,4 \%)$.

El efecto del CMBS sobre el pronóstico de la enfermedad es inconsistente en las series. La diferencia en estos resultados puede ser por el bajo número de pacientes reportados, y diferencias en la definición de los criterios diagnósticos, tipo de pacientes y seguimiento. Esta misma controversia ocurre cuando se comparan pacientes con tumores bilaterales sincrónicos y metacrónicos ${ }^{2,4}$. A pesar de ser un grupo más joven, al comparar los resultados de sobrevida, las pacientes con CMBS cursan con menor sobrevida, que nuestra cohorte control, sin embargo, esta diferencia no es estadísticamente significativa.

El tamaño limitado del subgrupo con CMBS impide comparaciones entre subgrupos y nuestros resultados con más bien informativos.

\section{Conclusiones}

El CMBS es poco frecuente. Usualmente son tumores RE positivos, donde el tumor contra lateral generalmente es pesquisado por imágenes, lo que resalta la importancia de la evaluación de la mama opuesta. Estos tumores son manejados menos frecuentemente con cirugía conservadora y aparentemente cursan con menor sobrevida.

\section{Referencias}

1. http://www.ine.cl. Estadísticas Vitales. 2009. Visitado el 12-05-2013.

2. Kheirelseid EA, Jumustafa H, Miller N, Curran C, Sweeney K, Malone C, et al. Bilateral breast cancer: analysis of incidence, outcome, survival and disease characteristics. Breast Cancer Research and Treatment 2011;126:131-40.

3. Hartman M, Czene K, Reilly M, Adolfsson J, Bergh J, Adami H-O, et al. Incidence and prognosis of synchronous and metachronous bilateral breast cancer. Journal of Clinical Oncology 2007;25:4210-6.

4. Schmid SM, Pfefferkorn C, Myrick ME, Viehl CT, Obermann E, Schötzau A, et al. Prognosis of early-stage synchronous bilateral invasive breast cancer. European Journal of Surgical Oncology 2011;37:623-8.

5. Elston CW, Ellis IO. Pathological prognostic factors in breast cancer. I. The value of histological grade in breast cancer: experience from a large study with long-term follow up. Histopathology 1991;19:403-10.

6. Goldhirsch A, Wood WC, Coates A S, Gelber RD, Thürlimann B, Senn H-J. Strategies for subtypes-dealing with the diversity of breast cancer: highlights of the St. Gallen International Expert Consensus on the Primary Therapy of Early Breast Cancer 2011. Annals of Oncology 2011;22:1736-47.

7. Shi Y-X, Xia Q, Peng R-J, Yuan Z-Y, Wang S-S, An X, et al. Comparison of clinicopathological characteristics and prognoses between bilateral and unilateral breast cancer. Journal of Cancer Research and Clinical Oncology 2012;138:705-14.

8. Filippakis GM, Georgiadou D, Pararas N, Lanitis S, Zografos G. Synchronous bilateral breast cancer with different biological profile and estrogen-progesterone receptor status. The Breast Journal 2008;14:209-10.

9. Sutcliffe JB, Otto PM. Controversies in breast MRI. Current Problems in Diagnostic Radiology 2013;42:14963.

10. Lehman C, Gatsonis C, Kuhl CK, Hendrick RE, Pisano ED, Hanna L, et al. MRI Evaluation of the Contralateral Breast in Women with Recently Diagnosed Breast Cancer. N Engl J Med 2007;356:1295-303.

11. Nichol AM, Yerushalmi R, Tyldesley S, Lesperance M, Bajdik CD, Speers C, et al. A case-match study comparing unilateral with synchronous bilateral breast cancer outcomes. Journal of Clinical Oncology 2011;29:47638.

12. Lee MM, Heimann R, Powers C, Weichselbaum RR, Chen LM. Efficacy of Breast Conservation Therapy in Early Stage Bilateral Breast Cancer. The Breast Journal. 1999;5:36-41. 